\title{
Critical review in qigong \& immunity cancer research
}

\begin{abstract}
Qigong, a common form of "Breathing and Relaxation Exercise", has been adopted by many Asian cancer patients as a complementary treatment in parallel with their conventional cancer management. Research has been conducted in many different centres not limited to Asian countries and reported in many scientific literatures. Most of these studies found short-term Qigong is able to achieve statistically significant clinical benefits as reported in cancer patients' self-reported quality of life including relieving chronic fatigue and many other psychological parameters. However there is limited unequivocal evidence on the clinical effects on the precise mechanism through which Qigong is able to achieve an overall general health enhancement for cancer patients. This mini-review aims to summarize the published specific immunological data about the innate as well as the adaptive anti-cancer immune bioactivities for cancer patients who practice Qigong in comparison with various control groups. We aim to relate the various biological components of the changes of specific immune populations including their number and activities, with the marked changes of the cytokine profiles as well as the circulating blood inflammation markers expression. This paper addresses the current research gap in the lack of sophisticated understanding of the mechanistic of Qigong leading to enhanced immunity. Furthermore, this review provides a map of future global collaborative efforts in conducting carefully design randomised control trials on selected commonly used Qigong exercises in cancer management.
\end{abstract}

Volume 7 Issue 3 - 2017

\author{
Daniel Man-yuen SZE,' Vincent Chan,' Maria \\ Benxia Wu,' Jing Xu,' Alex Chi keung $\mathrm{Ng}^{2}$ \\ 'School of Health and Biomedical Sciences, RMIT University, \\ Australia \\ ${ }^{2}$ Federation University, Australia
}

Correspondence: Daniel Man-yuen SZE, School of Health and Biomedical Sciences, RMIT University, PO Box 7I, Bundoora,Vic 3083 Australia, Tel +6I 39925 6524,

Email daniel.sze@rmit.edu.au

Received: April 05, 2017| Published: June 08, 2017

Keywords: qigong, qi, bre, cancer, immunology

Abbreviations: BRE, breathing relaxation exercise; QOL, quality of life; CRP, c-reactive protein; MQ, medical qigong; MicroRNA, micro ribonucleic acid; TCE, tai chi easy; WBC, white blood cell; RBC, red blood cell; DC, dendritic cell; IFN $\gamma$, interferongamma; TNF $\alpha$, tumour necrosis factor alpha; IL, interleukin; PHA, phytohaemagglutinin; $\mathrm{CD}$, cluster of differentiation protein; NK, natural killer cells

\section{Introduction}

Qigong is a general term for a number of different traditional Chinese energy exercises which have been practiced by millions of people in China for thousands of years. Research studies have shown that the practicing of Qigong has the following benefits of: Decreased heart rate; ${ }^{1}$ Decreased blood pressure; ${ }^{2}$ Lowered lipid levels; ${ }^{3}$ and Decreased levels of circulating stress hormones. ${ }^{4}$

Qigong, same as the other Breathing and Relaxation Exercises, consists of three major components: physical exercise, breathing, and meditation. The study conducted by Larkey et al., ${ }^{5}$ aimed to examine the differences between these three components together versus physical exercise alone for the clinical beneficial effects of Qigong using a double-blind, randomized controlled trial over 12weeks of Qigong /TCE versus Sham Qigong on fatigue, depression, and sleep among 87 postmenopausal, fatigued breast cancer survivors, stages 0 III, age 40-75. The researchers concluded that gentle exercise - that is, simply getting breast cancer survivors moving - may be beneficial in reducing a number of symptoms and boosting well-being of breast cancer survivors (depression and sleep quality). Furthermore, Qigong with breath/meditation focus has the advantage of improving one of the most recalcitrant symptoms for these survivors - persistent fatigue.

Based on the Traditional Chinese Medicine theory, Qigong is considered to be able to strengthen or balance the subtle energy (qi) circulating throughout a person's entire body, achieve the optimal wellbeing of harmonising the body, mind and spirit of a person, and thus improve overall health and fence off diseases. It has been postulated that while blockage or stagnation of energy flow in the energy channel in the human body will lead to discomfort, pain and sickness; then a restoration of a free flow and balance energy will lead to health improvement or even prevention of diseases. ${ }^{6}$

There are many comprehensive studies showing Qigong can improve a person's overall health conditions. These psychological and physiological aspects include bone density, cardiopulmonary effects, physical function, falls, balance and related risk factors, QOL, self-efficacy, anxiety, depression, immunity, and inflammation-related responses. $^{7-10}$

For instance, according to Wang et al., ${ }^{9}$ a meta-analysis on 3 studies of Diabetes type 2 patients suggested that Qigong was effective in reducing depression, and anxiety, and in improving psychological well-being. However, the researchers have also pointed out that due to the limitation of the research methodology, these studies were not able to dissect the contribution of Qigong alone from the effects of the other components of BRE such as peer learning, social support and positive expectations - collectively leading to the overall beneficial psychological wellbeing after the Qigong practice.

In relation to cancer treatment, there has been many systematic reviews conducted studying the benefits of Qigong for cancer patients. ${ }^{11-15}$ Many studies have shown that Qigong is able to offer a variety of benefits to cancer patients such as improvements in sleep, fatigue and QOL 6,16,17 and improvements in mood status and reduce specific side-effects of treatment. ${ }^{6}$

Recurring concluding remarks in many reviews examining the multiple completed QOL studies echo the inherent problems of research methodological limitations and small sample sizes. For 
instance, Oh et al., ${ }^{18}$ urged the need of more robust evidence in the form of randomised controlled trials with larger sample sizes.

Another important gap of clear evidence is the lack of a complete mechanistic understanding of how Qigong works because of little quantitative and objective measurements in most of the studies reported so far, with the exception of the inflammation biomarker CRP. In a 162-patient randomised clinical trial study conducted in Australia and USA, CRP expression was statistically significantly down regulated in the blood from patients who completed a 10-week Medical Qigong ${ }^{\mathrm{MQ}}$ program in comparison with the control group who had not participated in a program. ${ }^{6}$

\section{Discussion}

In this section we aim to consolidate some published data quantitative and objective about how Qigong impacts on cancer management. These areas include cancer patients' overall survival data; and effects of blood immune populations and cytokine profiles in healthy subjects and cancer patients respectively. We then relate these findings with the current knowledge of our body's anti-cancer natural immunity mechanisms. With these understanding, this minireview paper also aims to draw an overall picture to inform future research directions for evidence-based medicine of using Qigong in cancer management.

\section{Cancer patients' overall survival data}

We have summarized three of the survival studies of Qigong patients in Table 1. One of the studies showed that $17 \%$ of the lung cancer patients who practiced Qigong were able to attain a 5 -year survival rate, while the non-Qigong patients attained a 7\% 5-year survival rate only; ${ }^{19}$ while another study showed $30 \%$ of the Qigong patients attained the 5-year survival rate. ${ }^{20}$ Yet, another study highlighted that cancer patients who received Qigong exercise plus operation and herbal medicine were able to achieve a median survival period of 48 months as compare to another group of patients who only received operation and herbal medicine achieving a median survival period of 36.5months only. ${ }^{21}$ This implies Qigong exercise is able to achieve a minimum $30 \%$ improvement in both cases. However, all these studies were not able to provide a biomedical explanation as to what caused these results. Therefore, it is of high priority for researchers to understand the biological mechanisms of the human body, in particular how and why Qigong can achieve prolonged survival rate for cancer patients.

Table I Survival Table of Qigong Cancer Patients in 3 selected studies

\begin{tabular}{|c|c|c|c|c|c|}
\hline Reference & study design & $\begin{array}{l}\text { Cancer type \& } \\
\text { sample size }\end{array}$ & Qigong intervention & Measure(s) & $\begin{array}{l}\text { Key findings of the beneficial effects } \\
\text { of qigong exercise on survival }\end{array}$ \\
\hline \multirow{3}{*}{ Fu et. al. ${ }^{21}$} & \multirow{3}{*}{$\begin{array}{l}\text { Randomised } \mathrm{CT} \\
\mathrm{N}=186 \text { Four } \\
\text { groups }\end{array}$} & \multirow{3}{*}{$\begin{array}{l}\text { Gastric adenocar- } \\
\text { cinoma }\end{array}$} & & \multirow{3}{*}{$\begin{array}{l}\text { Survival rate in years } \\
\text { I, } 3 \text { and } 5 \text { years; } \\
\text { Median survival } \\
\text { period }\end{array}$} & $\begin{array}{l}\text { - Gp I: } 80 \%, 38 \%, 21 \% \text {; Median } \\
\text { Survival: } 36 \text { months }\end{array}$ \\
\hline & & & $\begin{array}{l}\text { All groups had operation } \\
\text { Gp I: no further treatment } \\
\text { Gp 2: chemo only } \\
\text { Gp 3: herbal only } \\
\text { Gp 4: herbal plus qigong }\end{array}$ & & $\begin{array}{l}\text { - Gp 2: } 86 \%, 45 \%, 23 \% \text {; Median } \\
\text { Survival: } 37 \text { months } \\
\text { - Gp 3: } 85 \%, 44 \%, 26 \% \text {; Median } \\
\text { Survival: } 30 \text { months }\end{array}$ \\
\hline & & & & & $\begin{array}{l}\text { - Gp } 4: 86 \%, 64 \%, 34 \% \text {; Median } \\
\text { Survival: } 48 \text { months }\end{array}$ \\
\hline \multirow[t]{2}{*}{ Zhang et. al. ${ }^{20}$} & $\begin{array}{l}\text { Not randomised; } \\
\mathrm{N}=\mathrm{No} \text { control CT } \\
\mathrm{N}=\mathrm{C48}\end{array}$ & $\begin{array}{l}\text { Various advanced } \\
\text { cancers }\end{array}$ & $\begin{array}{l}\text { Self-control Qigong with } \\
\text { standard treatment for } \\
\text { advanced cancer patients for } \\
8 \text { years }\end{array}$ & Survival rate 5 years & - More than $30 \%$ survived \\
\hline & \multirow{3}{*}{$\begin{array}{l}\text { Controlled CT, } \\
\text { al. }{ }^{19} \text { not randomised } \\
\mathrm{N}=100\end{array}$} & \multirow{3}{*}{$\begin{array}{l}\text { Various advanced } \\
\text { cancers including } \\
\text { liver, lung and } \\
\text { gastric }\end{array}$} & \multirow{3}{*}{$\begin{array}{l}\text { Qigong group versus No } \\
\text { Qigong group }\end{array}$} & \multirow{3}{*}{$\begin{array}{l}\text { Survival rate I and } \\
5 \text { years for lung and } \\
\text { gastric cancers; } \\
\text { Median survival } \\
\text { period for liver } \\
\text { cancer }\end{array}$} & $\begin{array}{l}\text { - Lung cancer: Qigong group I- and } \\
5 \text {-year survival rates were } 83 \% \text { and } \\
\text { I7\% (Control Group 5-year of } 7 \% \text { ) }\end{array}$ \\
\hline Zheng et. al. ${ }^{19}$ & & & & & $\begin{array}{l}\text { - Gastric cancer: Qigong group I- } \\
\text { and 5-year survival rates were } 83 \% \\
\text { and } 23 \% \text { (Control Group 5-year } \\
\text { of } 12 \% \text { ) }\end{array}$ \\
\hline & & & & & $\begin{array}{l}\text { - Liver cancer: Qigong group median } \\
\text { survival period was } 20.7 \text { months } \\
\% \text { (Control Group of } 3.5 \text { months } \\
p<0.0 \text { I) }\end{array}$ \\
\hline
\end{tabular}

\section{Effects of blood immune populations and cytokine profiles in healthy subjects}

The effect of Qigong in enhancing human immune functions has been widely studied. ${ }^{22-24}$ Ryu et al., ${ }^{22}$ reported an increase in T-lymphocytes while Lee et al., ${ }^{23}$ reported that the growth hormone (GH) concentrations and $\mathrm{O} 2-$ production by neutrophils (PMNs) was significantly increased for Qigong practitioners as compared to the base group. On the other hand, Manzaneque et al., ${ }^{24}$ have found lower number of total WBC and eosinophil numbers and \% of monocytes, as well as $\mathrm{C} 3$ complement concentration and a lower number of leukocytes in the Qigong group. The lower leukocyte counts also consisted, specifically, of a reduction of phagocytic cells.

This finding of lower concentration of C3 complement and neutrophils supports the result of lower inflammation cases for the Qigong group as compared to the control group. This is also supported by the findings reported by $\mathrm{Oh}$ et al., ${ }^{6}$ examining the effects in cancer patients. 


\section{Effects of blood immune populations and cytokine profiles in cancer patients}

Oh et al. ${ }^{6,17}$ reported that the use of a 10-week practice of MQ intervention in comparison with the control group has demonstrated statistically significant clinical benefits as shown in 162 cancer patients' self-reported chronic fatigue, QOL, and reduction of the inflammatory marker CRP in blood samples. However, the researchers also admitted that they did not find a significant association between CRP and self-reported chronic fatigue. Also, we believe the 10-week duration of Qigong practice is insufficient to reflect the trend how Qigong lowers the inflammatory marker of CRP as used by Oh et al., and its ability in relieving persistent fatigue $;^{5}$ therefore future studies of longer-term systematic Qigong practice periods are warranted.

The need for objective measures of the therapeutic mechanism of how Qigong has positive effects on decreasing inflammatory markers of CRP and cortisol is further reinforced by Zeng et al., ${ }^{12}$

In the relationship between Qigong and WBC count, Yeh et al., ${ }^{25}$ have found Qigong has significant benefits on reducing the side effects of chemotherapeutic treatments. After 3weeks of ChanChuang Qigong exercise, although there is no difference found in the RBC count, the WBC count is even higher than before treatment. After finishing the first 21-day chemotherapy, the average of WBCs or the Qigong group increased by 7\%, while the Control group decreased by $13 \%$ in comparison with initial WBC number at the beginning of this 3 -week duration. There is a significant difference between these 2 groups $(\mathrm{t}=3.18, \mathrm{P}<0.01)$.

Considering the impact of Qigong and the circulating blood cytokines, Jones ${ }^{26}$ examined the changes of the expression of 2 types of cytokines: Type 1 cytokines, such as IFN $\gamma$, TNF $\alpha$ and IL-12 which promotes cell-mediated responses; versus Type 2 cytokines, including IL-4, IL-6 and IL-10 which are anti-inflammatory and favour humoral responses. Jones ${ }^{26}$ reported that cancer patients practicing Guolin Qigong have significantly higher ratio of IFN $\gamma:$ IL-10 in PHAstimulated cultures at 3,7 and 14weeks than before training.

The importance of this up regulation of IFN $\gamma$ and downregulation of IL-10 in cancer patients who practice Guolin Qigong can be explained by relating to findings of cytokines that cause the dendritic cells (DCs) dysfunctioning versus restoration of the DC functionality as reported by Brown et al., ${ }^{27,28}$ Our laboratory has previously shown that Multiple Myeloma patients have reduced adaptive immunity as evidenced by their defective blood dendritic cells which have reduced co-stimulatory surface molecules of CD80 functionalities. Since DCs can be considered as the most pivotal antigen presenting cell type for the priming and activation of cancer-specific T cells, ${ }^{27}$ therefore such CD80 down regulation due to the presence of high level of IL10 and transforming growth factor $\beta 1$ (TGF $\beta$ ) in cancer patients can provide the explanation of patients' progressive deteriorating natural immunity.

Our research group then showed in subsequent experiments that the functionalities of these defective DCs in cancer patients can be restored by either IL-12 or IFN $\gamma .{ }^{27,28}$ Therefore an increased IFN $\gamma$ : IL10 ratio from cancer patients who practice Guolin Qigong can be taken as initial evidence of enhanced adaptive immunity in relation to Qigong practice for cancer patients.

In relation to the activation of NK cells which are one of the most important cell types in our body's innate immunity, Gruber et al., ${ }^{29}$ asserted that even though the number of CD56 \& NK cells has not changed, the NK cell activity increased as indicated by increase in the secretion of IFN $\gamma$. Cai et al., ${ }^{30}$ concurred with Gruber et al., ${ }^{19}$ that Qigong exercise can improve the WBC, CD20, IL-2 and NK activities. Wan ${ }^{31}$ has shown that the status of psychological state of cancer patients can exert a significant effect. The researchers showed that the ratio of $\mathrm{CD} 4 / \mathrm{CD} 8$ and $\mathrm{NK}$ cell number are closely related with depression in digestive cancer patients.

Collectively, these evidence shows that Qigong may enhance both the patients' innate immunity via the increased NK cell activities as well as restoration of the partially defective adaptive immunity through the enhancement of co stimulatory functionality of the specific DC subsets. These immunity enhancements may be partially explained by the change of patients' expression profiles of blood cytokines that may act as important messengers or even cross-talk mediators in these two immune pathways. Therefore it will be important in future studies to carefully assess the blood cytokine profiles, in particular the levels of IL-10 \& TGF- $\beta$ versus IL-12 and IFN $\gamma$, for the patients before and after the Qigong programs.

\section{Conclusion}

Based on our assertions made in the above, we see an urgent need for scientific Qigong and immunity cancer research which aims to address the gap in the lack of understanding of the underlying bioactivity mechanism of actions achieved by Qigong exercise through a global collaborative systematic evidence-based scientific study.

According to Lee et al., ${ }^{11}$ most of the controlled clinical trials on Qigong for cancer treatment suffered generally poor and greatly varied methodological quality. They also found two trials suggested effectiveness in prolonging life of cancer patients; but another one failed to do so. The effectiveness of Qigong in cancer care is not yet supported by the evidence from large scale carefully designed randomised clinical trials in multiple centres.

Therefore, we plan to conduct randomised control trials on selected commonly used Qigong in cancer management to provide high quality quantitative data derived from rigorous clinical observations supported by mechanistic laboratory investigations. Future research should be designed to meet the need for more rigorous clinical trials to confirm how Qigong can improve cancer patients' immune functions and to examine the possible underlying mechanisms of how it improves the immune function of cancer patients.

In addition, we will aim to study the patient's overall survival data and the improvement of patients' QOL through the collaborative development of a robust QOL survey. Furthermore, we will validate our hypothesis by studying the changes in patients' innate immunity (i.e. measuring the number and functionality of various blood NK subsets), changes in patients' adaptive immunity (i.e. measuring the number and functionality of various blood DC subsets and monoclonal $\mathrm{T}$ cells), as well as changes in the blood cytokine profile (in particular the levels of IL-10 \& TGF $\beta$ versus IL-12 and IFN $\gamma$ ). Furthermore, future studies also seek evidence of Qigong in relation to the changes of the blood Micro-RNA profiles that affect the summative micro RNAs produced from various immunological populations in blood not limited to the NK cells and DCs mentioned above.

\section{Acknowledgments}

None.

\section{Conflicts of interest}

Author declares there are no conflicts of interest. 


\section{Funding}

None.

\section{References}

1. Lee MS, Kim MK, Lee YH. Effects of Qi-therapy (external Qigong) on cardiac autonomic tone: a randomized placebo controlled study. Int $J$ Neurosci. 2005;115(9):1345-1350.

2. Lee MS, Lee MS, Kim HJ, et al. Qigong reduced blood pressure and catecholamine levels of patients with essential hypertension. Int $J$ Neurosci. 2003;113(12):1691-1701.

3. Lee MS, Lee MS, Kim HJ, et al. Effects of qigong on blood pressure, high-density lipoprotein cholesterol and other lipid levels in essential hypertension patients. Int J Neurosci. 2004;114(7):777-786.

4. Ryu H, Lee HS, Shin YS, et al. Acute effect of qigong training on stress hormonal levels in man. Am J Chin Med . 1996;24(02):193-198.

5. Larkey LK, Roe DJ, Weihs KL, et al. Randomized controlled trial of Qigong/Tai Chi Easy on cancer-related fatigue in breast cancer survivors. Ann Behav Med. 2015;49(2):165-176.

6. Oh B, Butow P, Mullan B, et al. Impact of medical Qigong on quality of life, fatigue, mood and inflammation in cancer patients: a randomized controlled trial. Ann Oncol. 2010;21(3):608-614.

7. Jahnke R, Larkey L, Rogers C, et al. A comprehensive review of health benefits of qigong and tai chi. Am J Health Promot. 2010;24(6):e1-e25.

8. Yang Y, Verkuilen J, Rosengren KS, et al. Effects of a Taiji and Qigong intervention on the antibody response to influenza vaccine in older adults. Am J Chin Med. 2007;35(04):597-607.

9. Wang F, Man JK, Lee EK, et al. The effects of qigong on anxiety, depression, and psychological well-being: a systematic review and metaanalysis. Evid Based Complement Alternat Med. 2013;2013:152738.

10. Oh B, Choi SM, Inamori A, et al.Effects of qigong on depression: a systemic review. Evid Based Complement Alternat Med 2013;2013:134737.

11. Lee MS, Chen KW, Sancier KM, et al. Qigong for cancer treatment: a systematic review of controlled clinical trials. Acta Oncol. 2007;46(6):717-722.

12. Zeng Y, Luo T, Xie H, et al. Health benefits of qigong or tai chi for cancer patients: a systematic review and meta-analyses. Complement Ther Med. 2014;22(1):173-186.

13. Chen K, Yeung R. Exploratory studies of qigong therapy for cancer in China. Integr Cancer Ther. 2002;1(4):345-370.

14. Chen K, Yeung R. A review of qigong therapy for cancer treatment. Journal-International Society of Life Information Science. 2002;20(2):532-542.

15. Chan CL, Wang CW, Ho RT, et al. A systematic review of the effectiveness of qigong exercise in supportive cancer care. Supportive Care Cancer. 2012;20(6):1121-1133.

16. Liu W, Schaffer L, Herrs N, et al.Improved sleep after Qigong exercise in breast cancer survivors: A pilot study. Asia Pac J Oncol Nurs. 2015;2(4):232-239.
17. Oh B, Butow PN, Mullan BA, et al. Effect of medical Qigong on cognitive function, quality of life, and a biomarker of inflammation in cancer patients: a randomized controlled trial. Supportive Care Cancer. 2012;20(6):1235-1242.

18. Oh B, Butow P, Mullan B, et al. A critical review of the effects of medical Qigong on quality of life, immune function, and survival in cancer patients. Integr Cancer Ther. 2012;11(2):101-110.

19. Zheng R. Observation of 100 cases with comprehensive qigong therapy for treating later-stage cancer. World Qigong. 1990;3(19):198.

20. Zhang X. Chinese Encyclopedia of Qigong [in Chinese]: Beijing. 1995.

21. Fu JZ, Fu SL, Qin JT. Effect of qigong and anticancer bodybuilding herbs on the prognosis of postoperative patients with cardiac adenocarcinoma. Third World Conference on Medical Qigong, China. 1996.

22. Ryu H, Jun CD, Lee BS, et al. Effect of qigong training on proportions of T lymphocyte subsets in human peripheral blood. Am J Chin Med. 1995;23(01):27-36.

23. Lee, M.S., M.K. Kim, and H. Ryu. Qi-training (qigong) enhanced immune functions: what is the underlying mechanism? Int J Neurosci. 2005;115(8): 1099-1104.

24. Manzaneque, J.M., F.M. Vera, et. al. Assessment of immunological parameters following a qigong training program. Medical Science Monitor. 2004;10(6):CR264-CR270.

25. Yeh ML, Lee TI, Chen HH,et al. The influences of Chan-Chuang qigong therapy on complete blood cell counts in breast cancer patients treated with chemotherapy. Cancer Nurs. 2006;29(2):149-155.

26. Jones BM. Changes in cytokine production in healthy subjects practicing Guolin Qigong: a pilot study. BMC Complement Altern Med . 2001;1(1):8

27. Brown RD, Pope B, Murray A, et al. Dendritic cells from patients with myeloma are numerically normal but functionally defective as they fail to up-regulate CD80 (B7-1) expression after huCD40LT stimulation because of inhibition by transforming growth factor- $\beta 1$ and interleukin-10. Blood. 2001;98(10):2992-2998.

28. Brown, R., A. Murray, B. Pope, et. al. Either interleukin-12 or interferon- $\gamma$ can correct the dendritic cell defect induced by transforming growth factor $\beta 1$ in patients with myeloma. Br J Haematol. 2004;125(6):743-748.

29. Gruber BL, Hersh SP, Hall NR, et al. Immunological responses of breast cancer patients to behavioral interventions. Biofeedback and Selfregulation. 1993;18(1):1-22.

30. Cai G, Zhang Y, Zhu Q, et al. The changes of multiple immune indicators after Guo-Lin Qigong practice among cancer patients. Eighth International Symposium on Qigong, China. 2001.

31. Wang W, Wu Y, Zhao Y, Effect of Guolin Qigong on psychological states and the immune functioning of lung cancer patients. The Relevance of the Wisdom Traditions in Contemporary Society: The Challenge to Psychology, China. 2004;pp:209. 